\title{
Nonlinear Cascade Strategy for Longitudinal Control in Automated Vehicle Guidance
}

\author{
Rachid ATTIA, Rodolfo ORJUELA and Michel BASSET \\ Modelling Intelligence Process and Systems (MIPS) laboratory, EA2332 \\ Université de Haute-Alsace, 12 rue des frères Lumière \\ F-68093 Mulhouse Cedex, France. \\ E-mail : rachid.attia@uha.fr - Fax : +33 389423282
}

\begin{abstract}
This paper deals with automatic control design for automotive driving with a special focus on the longitudinal control. The automotive vehicle is a complex system characterised by highly nonlinear longitudinal and lateral coupled dynamics. Consequently, the control design for automated driving should deal with both of these dynamic couplings. Indeed, the longitudinal control plays an important role in the automated guidance to ensure safety and comfort of automotive passengers. In this work, a nonlinear cascade longitudinal control based on inner and outer-loops design is proposed. The lateral control is handled following a model predictive approach ensuring the automated steering of the vehicle. Finally, the nonlinear longitudinal control is integrated with the lateral control in a whole architecture to perform a coupled longitudinal and lateral control. The effectiveness of the automated driving strategy is highlighted through simulation results.
\end{abstract}

Keywords: Autonomous vehicle guidance, Longitudinal control, Lateral control, Nonlinear control, Model predictive control.

\section{Introduction}

During last years, the field of automotive vehicle is experiencing an important evolution due to an increasing use of individual vehicles in everyday life. This increasing use poses new challenges such as safety and comfort of car passengers, traffic management, reduction of fuel consumption and pollutant emissions. To cope with these challenges, several automatic systems for driver assistance, chassis stabilization and engine control have been developed by automotive manufacturers and academicians. An interesting way to investigate the bring of automatic 
control systems can be the automated driving framework. The automated driving offers an appropriate framework to develop and test new guidance architectures that may equip the vehicles in the future.

In this work, the proposed automated driving architecture is depicted in Figure 1 and can be synthesized into three main layers. The outer layer percepts, using exteroceptive sensors (GPS, camera, ...), the environment in which the vehicle evolves and provides the relevant information to the reference generation layer. The reference generation provides two kinds of reference profiles, geometric path and reference speed, required for control. The reference signals are computed from the information provided by the perception layer (see [1] and references therein). The control layer ensures the automated vehicle driving along the desired path at desired speed. This task is accomplished by providing the appropriate control signals i.e. action on throttle, brake and steering wheel. This paper deals with the control design for automated driving.

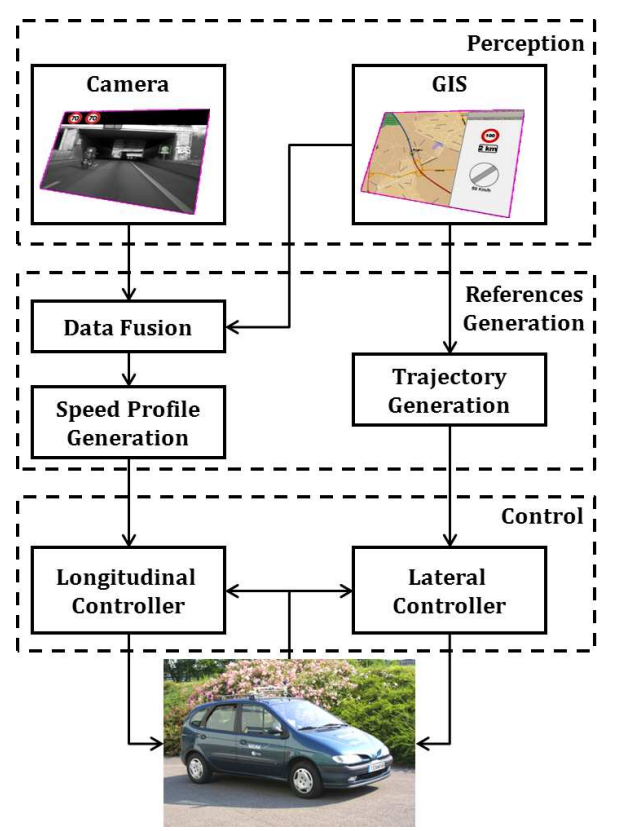

FIGURE 1: Automated vehicle guidance architecture.

The control law design for automated vehicle guidance is a non trivial problem due to the strong longitudinal and lateral couplings arising in the vehicle dynamics, for instance :

- Kinematic and dynamic coupling of the longitudinal and lateral motions due 
to the yaw motion caused by the steering of the front wheels.

- The load transfer phenomenon due to the longitudinal and lateral accelerations. These accelerations affect the tyre normal forces and so the longitudinal and lateral tyre forces.

- Tyre-road coupling forces constrained by the so-called friction circle/ellipse. In fact, the maximal available tyre-road friction is distributed between lateral and longitudinal forces.

Hence, the design of longitudinal, lateral and longitudinal/lateral control strategies becomes particularly arduous due to the strongly nonlinear models necessary to capture these couplings in a wide operational range [15]. Some authors attempt to consider simultaneously longitudinal and lateral couplings to obtain an only one controller ensuring coupled control goals. A sliding mode technique for coupled longitudinal and lateral control is proposed in [14] and recently a solution based on flatness control theory is presented in [20,21]. It can be emphasised that the main handled couplings are the dynamics coupling of the chassis motion. However, other dynamics should be considered (e.g. powertain dynamics), therefore increasing the control law design complexity. This complexity can be partially overcame following a decoupled lateral and longitudinal control synthesis under some assumptions as proposed in [2].

The lateral control can be investigated using different techniques. A fuzzy control approach is adopted in [17] to deal with this problem. The fuzzy controller is compared to a classic Lyapunov controller and shows effective performance. A neural network controller designed using genetic algorithms is employed in [23]. However, the stability proof and the performance analysis for artificial intelligence control are hard to be established. Recently, Model Predictive Control (MPC) has been explored to cope with this problem, see $[4,8,11]$ and references therein. In fact, the automatic steering of the vehicle is a complex constrained problem and MPC is a powerful tool which allows an intuitive handling of the constraints on both state and control inputs. The previously quoted papers have shown a successful implementation and have brought out the relevance of the MPC to cope with the lateral control.

The longitudinal control mainly deals with the development of new Active Cruise Control (ACC) which improves the classic Cruise Control (CC). The latter controller is designed for speed tracking, particularly in highway driving. However, some advanced applications such as autonomous vehicle guidance require high longitudinal vehicle capacities to track time varying speeds [24]. In fact, the reference speed is adapted according to the driving situations (highway, urban,...) thanks to perception and reference generation levels, see Figure 1. In [22], an ad- 
vanced longitudinal control design using sliding mode technique is proposed and experimentally validated. An ACC based on a gain-scheduling control technique is recently proposed in [28]. In the quoted papers, the authors attempt to consider the powertrain dynamics but the tyre-road interaction is neglected. Recently, a complex nonlinear model for longitudinal vehicle dynamics considering the tyreroad interaction to enhance the longitudinal control performance is proposed in [18]. A similar approach is proposed here considering also the powertrain dynamics.

The main purpose of this paper is to propose a global control strategy for automated vehicle guidance. A first issue of an integrated longitudinal and lateral guidance strategy taking into account safety aspects has been recently proposed by the authors in [2]. In that paper, the lateral control is based on a Nonlinear Model Predictive Controller (NMPC), the longitudinal control is ensured by a PI controller and the powertrain dynamics is not considered. The proposed PI longitudinal control is improved in [3] using a direct Lyapunov control method considering the powertrain dynamics but neglecting the tyre-road interaction. In this paper, the powertrain dynamics as well as the tyre-road interaction are handled. The whole longitudinal controller proposed here is based on a cascade control architecture for speed tracking (outer-loop) and torque control (inner-loop). The previously proposed NMPC for lateral control is replaced here by a linear MPC to reduce the computational effort. Finally, the longitudinal and lateral controllers are integrated in an interconnected architecture to perform automated driving.

The outline of this paper is as follows. The proposed nonlinear control design for longitudinal control is exposed in Section 2. Section 3 presents the linear MPC design and details the proposed coupled longitudinal and lateral control architecture. Simulation results are presented in Section 4. Section 5 wraps up the paper by a discussion on the role of longitudinal control in reducing fuel consumption.

\section{Longitudinal Control Strategy}

The goal of the longitudinal control is to ensure the tracking of time-varying reference speed. The whole longitudinal dynamics of the vehicle is characterized by a set of nested complex systems. An external system characterizes the relationship between the vehicle speed and the applied torque on the wheels. Its dynamics is governed by the longitudinal motion resulting from tyre-road contact forces. An internal system describes the interaction between the throttle and brake actions and the produced torque. This last implies complex elements such as engine, gearbox and driveline. 
The longitudinal control problem is tackled through a cascade control architecture depicted in Figure 2. The use of a cascade control has been motivated by the structure of the longitudinal dynamics. In this control architecture an outerloop ensures the reference speed tracking and calculates the torque to be applied on the wheels. An inner-loop provides the throttle opening and the brake pressure to generate the required control torque. In the forthcoming subsections both outer and inner-loops are designed and the whole control architecture is analysed.

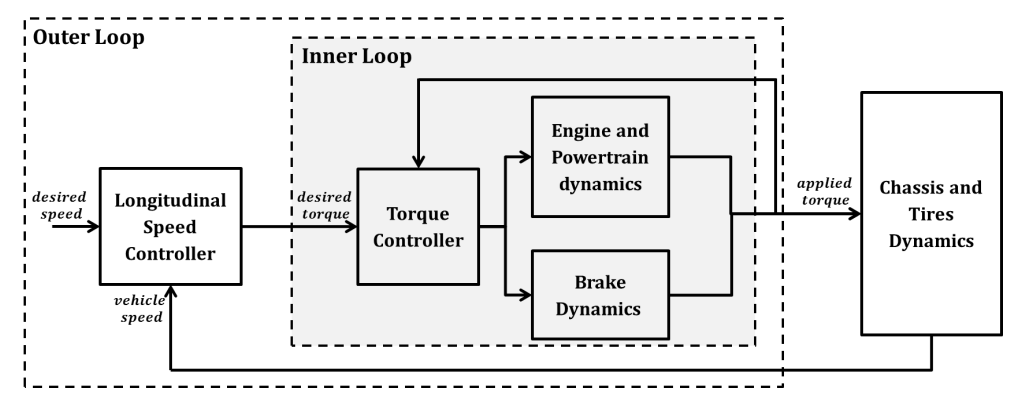

FIGURE 2: Longitudinal control architecture.

\subsection{Outer-loop Design for Reference Speed Tracking}

Consider a one-wheel vehicle model, the longitudinal motion of the vehicle is governed by [22] :

$$
m \dot{v}=F_{l}-C_{a} v^{2}-C_{r} m g \cos (\theta)-m g \sin (\theta)
$$

where $v$ is the vehicle speed, $m$ the vehicle mass, $F_{l}$ the traction force, $C_{a}$ the aerodynamic coefficient, $C_{r}$ the rolling resistance moment, $\theta$ the road slope and $g$ the gravitational acceleration. The traction force $F_{l}$ results from the tyre-road contact and acts on the wheel dynamics as following :

$$
I_{w} \dot{\omega}=-R F_{l}+T_{w}
$$

where $I_{w}$ is the wheel inertia, $\omega$ the wheel rotational speed, $R$ the wheel radius and $T_{w}$ the applied torque (traction or braking) on the wheel. The traction force $F_{l}$ results from the tyre-road contact and is given by :

$$
F_{l}=f_{\text {tyre }}\left(\lambda, \mu, F_{z}\right)
$$

where $f_{\text {tyre }}$ is a nonlinear function, $\lambda$ the longitudinal slip, $\mu$ the friction coefficient which depends on road conditions and $F_{z}$ the wheel vertical load. Several models of different complexity are proposed in the literature to characterise 
the nonlinear function $f_{\text {tyre }}$ according to the modelling purpose (see [12] for an overview on tyre modelling). In normal driving situations, i.e. moderate acceleration/deceleration $\left(<3 \mathrm{~ms}^{-2}\right)$, assuming a constant friction coefficient $\mu$ and a constant vertical load $F_{z}$, the friction force $F_{l}$ can be expressed by the following linear tyre model :

$$
F_{l}=C_{\lambda} \lambda
$$

where $C_{\lambda}$ is the longitudinal stiffness coefficient and $\lambda$ the longitudinal slip ratio given by :

$$
\lambda=\frac{R \omega-v}{\max (R \omega, v)}
$$

Let the longitudinal reference speed be a continuously differentiable signal $v_{r e f}$ and the tracking speed error defined by :

$$
e_{v}=v-v_{r e f}
$$

then, the tracking error dynamics is given by :

$$
\begin{aligned}
\dot{e}_{v} & =\frac{C_{\lambda}}{m} \lambda-\frac{C_{a}}{m} v^{2}-C_{r} g \cos (\theta)-g \sin (\theta)-\dot{v}_{r e f} \\
\dot{\omega} & =-\frac{R C_{\lambda}}{I_{w}} \lambda+\frac{T_{w}}{I_{w}}
\end{aligned}
$$

by considering (1), (2) and the longitudinal slip (5).

Remark that the use of a direct Lyapunov approach to stabilize the model (7) is not straightforward as shown in [18]. In fact, the speed tracking error (7a) and the wheel rotational speed (7b) are related through the longitudinal slip (5). Consequently, the stabilisation of (7a) is achieved here following a backstepping approach using a virtual control input $\lambda^{*}$. Then, the control input $T_{w}$ is calculated such as the longitudinal slip tracking error :

$$
e_{\lambda}=\lambda-\lambda^{*}
$$

converges towards zero. For that purpose, the longitudinal slip (5) is expressed differently in acceleration and deceleration phases.

In acceleration phase the longitudinal slip is expressed by :

$$
\lambda=1-\frac{v}{R \omega}
$$


and its time derivative by :

$$
\dot{\lambda}=-\left(\frac{R^{2}(1-\lambda)^{2}}{I_{w} v}+\frac{1-\lambda}{m v}\right) C_{\lambda} \lambda+\frac{R(1-\lambda)^{2}}{I_{w} v} T_{w}+\frac{1-\lambda}{m v} F_{r}(v)
$$

where $F_{r}$ gathers the different resistance forces :

$$
F_{r}(v)=\frac{C_{a}}{m} v^{2}+C_{r} g \cos (\theta)+g \sin (\theta)
$$

In deceleration phase the longitudinal slip is given by :

$$
\lambda=\frac{R \omega}{v}-1
$$

and its time-derivative :

$$
\dot{\lambda}=-\left(\frac{R^{2}}{I_{w} v}+\frac{1+\lambda}{m v}\right) C_{\lambda} \lambda+\frac{R}{I_{w} v} T_{w}+\frac{1+\lambda}{m v} F_{r}(v)
$$

It can be emphasised that the tyre remains in its linear operating range for a longitudinal slip ratio into the interval $\lambda \in[-0.07,0.1]$ as mentioned in [18]. Beyond these limits, the tires are saturated and the vehicle enters in pure slip motion. Taking into account these considerations, the following approximation can be considered :

$$
1-\lambda^{2}=(1-\lambda)(1+\lambda) \approx 1
$$

with :

$$
1-|\lambda|= \begin{cases}1-\lambda & \text { if } \lambda \geq 0 \\ 1+\lambda & \text { otherwise }\end{cases}
$$

Then, considering (14) and (15), the longitudinal slip dynamics in both acceleration and deceleration modes can be expressed, in a unified form, as :

$$
\dot{\lambda}=(1-|\lambda|)\left(-\left(\frac{R^{2}(1-\lambda)}{I_{w} v}+\frac{1}{m v}\right) C_{\lambda} \lambda+\frac{R(1-\lambda)}{I_{w} v} T_{w}+\frac{1}{m v} F_{r}(v)\right)
$$

and the longitudinal motion dynamics is finally rewritten using (7a) and (16) as follows :

$$
\begin{aligned}
\dot{e}_{v} & =\frac{C_{\lambda}}{m} \lambda-\frac{F_{r}(v)}{m}-\dot{v}_{r e f} \\
\dot{\lambda} & =(|\lambda|-1)\left(\left(\frac{R^{2}(1-\lambda)}{I_{w} v}+\frac{1}{m v}\right) C_{\lambda} \lambda-\frac{R(1-\lambda)}{I_{w} v} T_{w}-\frac{1}{m v} F_{r}(v)\right)(17
\end{aligned}
$$


The control design is achieved in two steps based on the direct Lyapunov approach. In the first step, the virtual control $\lambda^{*}$ is obtained to stabilise (17a) with the help of the following Lyapunov-candidate function :

$$
V_{1}=\frac{1}{2} e_{v}^{2}
$$

The exponential stability of the tracking error can be investigated satisfying the condition :

$$
\dot{V}_{1}=-2 k_{v} V_{1}
$$

where $k_{v}>0$ is a tuning parameter for convergence rate. The time derivative of equation (18) is given by :

$$
\dot{V}_{1}=e_{v} \dot{e}_{v}
$$

which becomes :

$$
\dot{V}_{1}=e_{v}\left(\frac{C_{\lambda}}{m} \lambda-\frac{F_{r}(v)}{m}-\dot{v}_{r e f}\right)
$$

and by using the stability condition (19), the following virtual control law is suggested:

$$
\lambda^{*}=\frac{m}{C_{\lambda}}\left(-k_{v} e_{v}+\frac{F_{r}(v)}{m}+\dot{v}_{r e f}\right)
$$

In the second step, the control torque $T_{w}$ is computed to stabilise the longitudinal speed tracking error (8) using a similar approach. The stabilizing control law is designed considering the Lyapunov-candidate function :

$$
V_{2}=\frac{1}{2} e_{\lambda}^{2}
$$

and the exponential stability condition :

$$
\dot{V}_{2}=-k_{\lambda} V_{2}
$$

where $k_{\lambda}>0$. The following stabilizing control law can be derived :

$$
\begin{aligned}
T_{w}^{*}= & \frac{I_{w} v}{R(1-|\lambda|)(1-\lambda)} \\
& \left(-k_{\lambda} e_{\lambda}-\frac{1-|\lambda|}{m v} F_{r}(v)+\frac{1-|\lambda|}{v}\left(\frac{R^{2}(1-\lambda)}{I_{w}}+\frac{1}{m}\right)+\dot{\lambda}^{*}\right)
\end{aligned}
$$


where

$$
\dot{\lambda}^{*}=\frac{m}{C_{\lambda}}\left(-k_{v}\left(\frac{C_{\lambda}}{m} e_{\lambda}-k_{v} e_{v}\right)+\frac{1}{m} \frac{\partial F_{r}(v)}{\partial v}\left(\frac{C_{\lambda}}{m} e_{\lambda}-k_{v} e_{v}+\dot{v}_{r e f}\right)+\ddot{v}_{r e f}\right)
$$

Finally, by considering the control laws (22) and (26), the closed loop dynamics is given by :

$$
\left[\begin{array}{c}
\dot{e}_{v} \\
\dot{e}_{\lambda}
\end{array}\right]=\left[\begin{array}{cc}
-k_{v} & \frac{C_{\lambda}}{m} \\
0 & -k_{\lambda}
\end{array}\right]\left[\begin{array}{l}
e_{v} \\
e_{\lambda}
\end{array}\right]
$$

Notice that, the closed loop dynamics (27) is stabilized for any strictly positive tuning parameters $k_{v}$ and $k_{\lambda}$. The convergence rate can be tuned using these parameters.

\subsection{Inner-loop Design for Torque Controller}

The inner-loop ensures the tracking of the torque reference $T_{w}^{*}$ provided by the outer-loop. The engine torque controller acts on the throttle input to track the required torque. The applied wheel torque is related to the throttle input through a complex nonlinear relationship due to the engine and the powertrain dynamics. The available engine torque depends on the throttle opening as well as the engine speed, this relationship is often given by lookup tables. The gearbox is also an important element in the powertrain dynamics and affects the engine behaviour through gear shifts. The gearbox introduces the discrete parameter, the gearbox ratio, which should be handled by the engine torque controller. As mentioned in [27], the engine and the powertrain dynamics can be modelled by :

$$
\begin{aligned}
I_{e} \dot{N}_{e} & =T_{e}\left(u_{t}, N_{e}\right)-\left(\frac{N_{e}}{K_{t c}}\right)^{2} \\
T_{w} & =R_{f} R_{t r} C_{t r}\left(\frac{N_{e}}{K_{t c}}\right)^{2}
\end{aligned}
$$

where $I_{e}$ and $N_{e}$ are the engine moment of inertia and speed, $K_{t c}$ the K-factor, $R_{f}$ the final drive ratio, $R_{t r}$ the gearbox ratio. The engine torque $T_{e}$ depends on the engine speed $N_{e}$ and the throttle opening $u_{t}$ following the relatioship [10] :

$$
T_{e}\left(u_{t}, N_{e}\right)=T_{e i}+\left(a_{0}+a_{1} N_{e}+a_{2} N_{e}^{2}\right) u_{t}
$$

where $T_{e i}$ is the engine torque when the throttle is closed. A characteristic of a gasoline engine is given in Figure 3. 


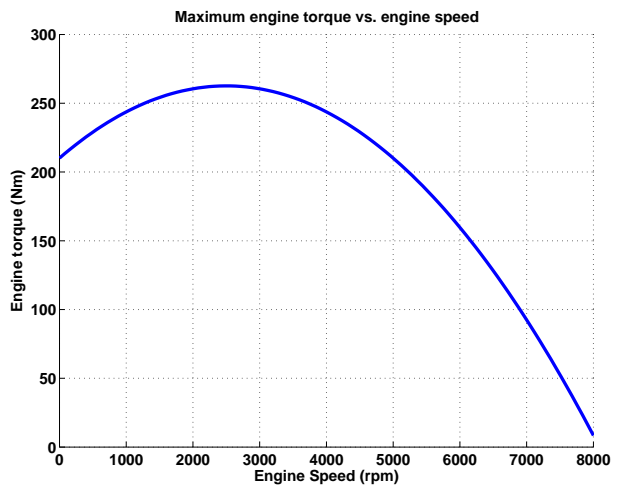

FIGURE 3: Engine map model.

In this paper, a gain scheduling PI control strategy is adopted to cope with the torque control. This approach allows to overcome the difficulties introduced by the discrete changes of the gear ratio. A set of PI controllers are synthesized considering the linearisation of the model (28) at different steady-state points $\left\{N_{e_{0}}, u_{t_{0}}=0\right\}$ corresponding to the different gear ratios :

$$
\begin{aligned}
I_{e} \Delta N_{e} & =-2 \frac{N_{e_{0}}}{K_{t c}^{2}} \Delta N_{e}+\left(a_{0}+a_{1} N_{e_{0}}+a_{2} N_{e_{0}}^{2}\right) u_{t} \\
T_{w} & =2 R_{f} R_{t r} C_{t r} \frac{N_{e}}{K_{t c}^{2}} \Delta N_{e}
\end{aligned}
$$

where $\Delta N_{e}=N_{e}-N_{e 0}$. The scheduling of this set of controllers is governed by the actual gearbox ratio. The stability analysis for this gain-scheduling strategy is not the main topic of the present work, see [26] for details on gain-scheduling design techniques.

\subsection{Stability of the Cascade Control Loop}

In the previous sections the inner and outer controllers are separately synthesised . Here, the behaviour of the cascade control is analysed assuming that the inner-loop is exponentially stable. Consequently, the following condition holds :

$$
\dot{e}_{T}=-k_{T} e_{T}
$$

where the torque tracking error is defined by :

$$
e_{T}=T_{w}-T_{w}^{*}
$$


with $T_{w}$ the actual torque and $T_{w}^{*}$ the desired torque. From (32), the impact of inner-loop dynamics on the cascade control can be considered as follows :

$$
T_{w}=T_{w}^{*}+e_{T}
$$

At this point, (33) and (17b) can be used to obtain :

$$
\dot{e}_{\lambda}=-k_{\lambda} e_{\lambda}+\frac{R(1-|\lambda|)(1-\lambda)}{I_{w} v} e_{T}
$$

Finally, using (27) and (34) the whole cascade loop dynamics is expressed by :

$$
\left[\begin{array}{c}
\dot{e}_{v} \\
\dot{e}_{\lambda} \\
\dot{e}_{T}
\end{array}\right]=\left[\begin{array}{ccc}
-k_{v} & \frac{C_{\lambda}}{m} & 0 \\
0 & -k_{\lambda} & \frac{R(1-|\lambda|)(1-\lambda)}{I_{w} v} \\
0 & 0 & -k_{T}
\end{array}\right]\left[\begin{array}{l}
e_{v} \\
e_{\lambda} \\
e_{T}
\end{array}\right]
$$

It is important to note that (35) is a Linear Parameter Varying (LPV) system. Therefore, the stability cannot be investigated based on the eigenvalues of the dynamic matrix due to the time-varying term :

$$
\rho=\frac{R(1-|\lambda|)(1-\lambda)}{I_{w} v}
$$

However, the stability can be established considering the bounded variation of the parameter $\rho \in[\rho, \bar{\rho}]$. Under this assumption, the time-varying term $\rho$ can be rewritten as follows :

$$
\rho=\rho_{1} \underline{\rho}+\rho_{2} \bar{\rho}
$$

where :

$$
\rho_{1}=\frac{\bar{\rho}-\rho}{\bar{\rho}-\underline{\rho}} \quad \text { and } \quad \rho_{2}=\frac{\rho-\underline{\rho}}{\bar{\rho}-\underline{\rho}}
$$

verifying the following convex properties :

$$
\sum_{i=1}^{2} \rho_{i}=1 \quad \text { and } \quad 0 \leq \rho_{i} \leq 1 \quad i=1,2
$$

Thanks to the proposed transformation (37), the equation (35) is rewritten as follows :

$$
\dot{e}=\sum_{i=1}^{2} \rho_{i} A_{i} e
$$


where :

$$
e=\left[\begin{array}{l}
e_{v} \\
e_{\lambda} \\
e_{T}
\end{array}\right], \quad A_{1}=\left[\begin{array}{ccc}
-k_{v} & \frac{C_{\lambda}}{m} & 0 \\
0 & -k_{\lambda} & \rho \\
0 & 0 & -k_{T}
\end{array}\right], \quad A_{2}=\left[\begin{array}{ccc}
-k_{v} & \frac{C_{\lambda}}{m} & 0 \\
0 & -k_{\lambda} & \bar{\rho} \\
0 & 0 & -k_{T}
\end{array}\right]
$$

Finally, the system (40) is exponentially stabilized if the following condition is satisfied [6] :

$$
A_{i}^{T} P+P A_{i}+2 \alpha P<0 \quad \text { for } \quad i=1,2
$$

where $\alpha$ is strictly positive scalar and $P$ a symmetric definite positive matrix of appropriate dimension to be found using Linear Matrix Inequality (LMI) solvers (see Section 4.2).

As far as here, it is assumed that the inner-loop is exponentially stable. However, the exponential stability is usually hard to obtain for the gain scheduling strategy. It can be interesting to analyse the stability when the tracking error of the inner-loop does not vanish but remains in a certain neighbourhood of the origin. For that purpose, the cascade dynamics equation (35) is modified as follows :

$$
\dot{e}=\sum_{i=1}^{2} \rho_{i} A_{i} e+B d
$$

where $B^{T}=\left[\begin{array}{lll}0 & 0 & 1\end{array}\right]$ and $d=d(t)$ is an energy bounded disturbance. In this case, an attenuation level of the disturbance effect on the tracking error vector $e$ can be expressed as follows :

$$
\frac{\|e\|_{2}^{2}}{\|d\|_{2}^{2}}<\gamma \quad \gamma>0
$$

where $\gamma$ is the attenuation level and $\|z\|_{2}^{2}$ the $\mathbb{L}_{2}$ norm of $z$ defined by :

$$
\|z\|_{2}^{2}=\int_{0}^{\infty} z^{T}(t) z(t) d t
$$

Both exponential stability and disturbance attenuation are met if the following condition holds [6] :

$$
\dot{V}<-2 \alpha V-e^{T} e+\gamma d^{T} d
$$

where $V$ is a Lyapunov-candidate function, for instance :

$$
V=e^{T} P e \quad P=P^{T}>0
$$


For a given $\alpha, A_{i}$ and $B$, the LMI formulation of the condition (44) is then expressed as [6] :

$$
\begin{aligned}
& \min _{P} \gamma \\
& {\left[\begin{array}{cc}
A_{i}^{T} P+P A_{i}+2 \alpha P+I & P B \\
B^{T} P & -\gamma I
\end{array}\right]<0 \text { for } i=1,2}
\end{aligned}
$$

where $I$ is the identity matrix of appropriate dimension. This LMI is not used to design the controller gains $k_{v}, k_{\lambda}$ and $k_{T}$ but only to investigate, a posteriori, closed-loop performance (i.e. decay rate and disturbance attenuation level). In fact, numerical solution of the LMI cannot be found for a high decay rate $\alpha$ and $\gamma<<1$, a trade-off between decay rate and attenuation level should be found.

\subsection{Automatic Gear Shift Management}

As mentioned above, the available control inputs are the throttle, the brake and the gearbox. A management policy should be defined to handle the working exclusivity between throttle and brake and a gear shift policy also has to be determined. The switching between throttle and brake is defined based on the throttle opening value given by the nonlinear torque controller and the speed tracking error. The brake is activated if the throttle is inactive and the vehicle speed is greater than the reference speed. The automatic gear shift management, i.e. determining the adequate gearbox ratio at each instant time, is a complex optimization problem [10] and will not be addressed in this work. However, several studies show that the optimal engine operating point for small road gradients is reached around $2750 \mathrm{rpm}$ [16]. From this observation, the solution adopted here results of an automatic gearbox-like system modelled by the statechart shown in Figure 4.

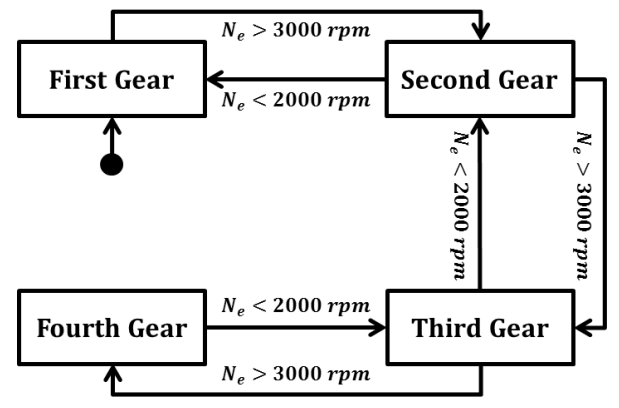

FIGURE 4: Gear shift strategy. 


\section{Combined Longitudinal and Lateral Control}

In this section, the lateral control design is presented and the whole guidance strategy is detailed.

\subsection{Lateral Control Strategy}

The lateral controller ensures the steering of the vehicle to follow a given geometric trajectory. For control design a linearised lateral model of the vehicle is shortly introduced. The lateral behaviour of the vehicle can be described in the linear operating range by the well-known bicycle model. The motion equations of the linear bicycle model depicted in Figure 5 are given by [12] :

$$
\left[\begin{array}{c}
\dot{v_{y}} \\
\dot{r} \\
\dot{Y} \\
\dot{\Psi}
\end{array}\right]=\left[\begin{array}{cccc}
\frac{C_{f}+C_{r}}{m v_{x}} & \frac{l_{r} C_{r}-l_{f} C_{f}}{m v_{x}}-v_{x} & 0 & 0 \\
\frac{l_{r} C_{r}-l_{f} C_{f}}{I_{z} v_{x}} & -\frac{l_{f}^{2} C_{f}+l_{r}^{2} C_{r}}{I_{z} v_{x}} & 0 & 0 \\
1 & 0 & 0 & 0 \\
0 & 1 & 0 & 0
\end{array}\right]\left[\begin{array}{c}
v_{y} \\
r \\
Y \\
\Psi
\end{array}\right]+\left[\begin{array}{c}
\frac{C_{f}}{m} \\
\frac{l_{f} C_{f}}{I_{z}} \\
0 \\
0
\end{array}\right] \delta_{f}
$$

where $v_{y}$ is the lateral speed, $r$ the yaw rate, $Y$ the lateral vehicle position, $\Psi$ the heading angle, $v_{x}$ the longitudinal vehicle speed, $\delta_{f}$ the steering angle, $l_{f}$ and $l_{r}$ the front and rear CoG-distance, $m$ the vehicle mass and $I_{z}$ the moment of inertia around the vertical. A linear tyre model (similar to (4)) is employed for lateral tyre forces modelling considering the front and rear cornering stiffness coefficients $C_{f}$ and $C_{r}$.

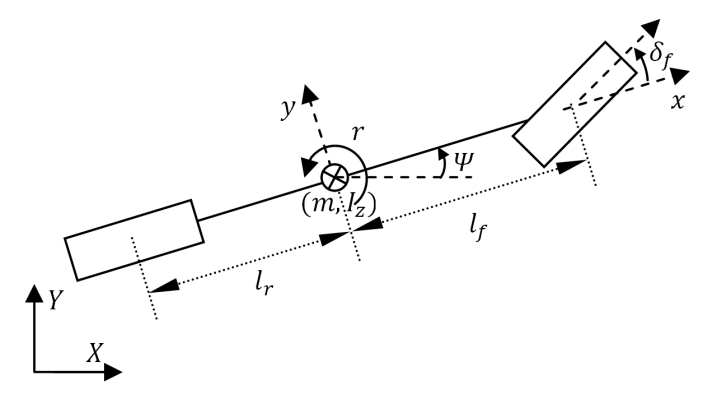

FIGURE 5: Bicycle model.

Notice that the linearised model of the vehicle (49) depends on the longitudinal speed $v_{x}$. The authors have proposed a Nonlinear Model Predictive Control (NMPC) to cope with the lateral control problem in a previous work [2]. The 
proposed approach is based on a nonlinear model capturing both of longitudinal and lateral dynamics and shows an effective trajectory tracking. However, the NMPC requires to solve a nonlinear optimization problem on-line and does not meet real-time constraints. In order to overcome this limitation, a Linear MPC is adopted here under the assumption of slowly varying longitudinal speeds.

The MPC allows reference tracking considering constraints on both control inputs and state variables [25]. The controlled inputs are calculated at each sampling time thanks to an on-line optimisation minimising the tracking errors between the predicted and the reference outputs on a prediction time-horizon $N_{p}$. The predicted outputs are obtained from a prediction model obtained from the discretisation of the continuous-time bicycle model (49). Euler's approximation is used for that purpose and the discrete-time model is given by :

$$
\begin{array}{ll}
\xi(k+1) & =A_{d} \xi(k)+B_{d} u(k) \\
h(k) & =C_{d} \xi(k)
\end{array}
$$

where $\xi=\left[\begin{array}{lll}v_{y} & Y \Psi\end{array}\right]^{T}$ and $h=\left[\begin{array}{l}Y \Psi\end{array}\right]^{T}$.

The cost function usually employed in the MPC optimisation problem is given by [25] :

$$
J=\sum_{n=1}^{N p}\left\|h(k+n \mid k)-h_{r e f}(k+n)\right\|_{Q}+\sum_{n=0}^{N c-1}\|u(k+n \mid k)\|_{R}
$$

where $h_{r e f}$ is the reference output and $N_{c}$ the control time-horizon which corresponds to the optimization problem dimension to be solved. Matrices $Q$ and $R$ respectively represent the weights regarding the tracking errors and the control input energy; the choice of these weight matrices is a classical issue in optimal and predictive control [19].

The $L M P C$ problem to cope with the lateral control is formulated as follows [2] :

$$
\begin{array}{ll} 
& \arg \min _{\Delta u} J(\xi(k), \Delta u) \\
\text { s.t } \quad & \xi(k+n+1 \mid k)=A_{d} \xi(k+n \mid k)+B_{d} u(k+n \mid k) \\
& h(k+n \mid k)=C_{d} \xi(k+n \mid k) \\
& u_{\min } \leq u(k+n \mid k) \leq u_{\max } \\
& \Delta u_{\min } \leq \Delta u(k+n \mid k) \leq \Delta u_{\max } \\
& u(k+n \mid k)=u(k+n-1 \mid k)+\Delta u(k+n \mid k) \\
& \Delta u(k+n \mid k)=0 \text { for } n=N_{c}, \ldots, N_{p}
\end{array}
$$


where $A_{d}, B_{d}$ and $C_{d}$ are already defined by (50). The notation $\xi(k+n \mid k)$ means that the predicted value of $\xi$ at instant $k+n$ such that $\xi(k \mid k)=\xi(k)$ i.e. at each sampling time the initial state for prediction is updated using the available measurements. Using the discrete-time model (50), the future states of the system are calculated following the expression :

$$
H=F \xi(k)+\Phi \Delta U
$$

where $H=\left[h(k+1 \mid k), \ldots, h\left(k+N_{p} \mid k\right)\right]^{T}, \Delta U=\left[u(k \mid k), \ldots, u\left(k+N_{c} \mid k\right)\right]^{T}$ and the matrices $F \in \mathbb{R}^{2 N_{p} \times 4}$ and $\Phi \in \mathbb{R}^{2 N_{p} \times N_{c}}$ given by :

$$
F=\left[\begin{array}{c}
C_{d} A_{d} \\
C_{d} A_{d}^{2} \\
\vdots \\
C_{d} A_{d}^{N_{p}}
\end{array}\right] \text { and } \Phi=\left[\begin{array}{ccc}
C_{d} B_{d} & 0 & 0 \\
C_{d} A_{d} B_{d} & C_{d} B_{d} & 0 \\
\vdots & & \\
C_{d} A_{d}^{N_{p}-1} B_{d} & \ldots & C_{d} A_{d}^{N_{p}-N_{c}} B_{d}
\end{array}\right]
$$

Consider (53) and define $H_{r e f}$ as the reference outputs for the prediction horizon then the optimization criterion (51) is written as follows :

$$
J=\left(H_{r e f}-H\right)^{T} Q\left(H_{r e f}-H\right)+\Delta U^{T} R \Delta U
$$

where $Q \in \mathbb{R}^{2 N_{p} \times 2 N_{p}}$ and $R \in \mathbb{R}^{N_{c} \times N_{c}}$ are the weights matrices. Finally, the optimization criterion (55) is written in a standard Linearly Constrained Quadratic Programming (LCQP) problem :

$J=\left(H_{r e f}-F \xi(t)\right)^{T} Q\left(H_{r e f}-F \xi(t)\right)-2 \Delta U^{T} \Phi^{T}\left(H_{r e f}-F \xi(t)\right)+\Delta U^{T}\left(\Phi^{T} \Phi R\right) \Delta U$

and the LCQP is then expressed as :

$$
\begin{aligned}
\arg \min _{\Delta u} J(\Delta U)= & \Delta U^{T}\left(\Phi^{T} \Phi R\right) \Delta U-2 \Delta U^{T} \Phi^{T}\left(H_{r e f}-F \xi(t)\right) \\
\text { s.t. } \quad & A_{e q} \Delta U \leq b_{e q}
\end{aligned}
$$

where $A_{e q}$ and $b_{e q}$ gather linear constraints on $\Delta U$.

\subsection{Longitudinal and Lateral Couplings}

The developed longitudinal and lateral controllers are integrated in a whole guidance strategy to ensure a coupled control. In fact, the lateral control acts as a disturbance on the longitudinal dynamics. However, the effect of the steering on the longitudinal speed tracking can be neglected in normal driving situations. On 
the other hand, the longitudinal speed tracking largely affects the lateral dynamics. Thus, the variations of the longitudinal speed should be considered in the coupled control approach as shown in Figure 6. In fact, the longitudinal speed $v_{x}$ acts on the lateral control such as an external disturbance. This interaction is given by the time-varying parameter $v_{x}$ in the bicycle model (49). Several MPC algorithms for Linear Parameter Varying (LPV) systems are proposed in the literature. In [5] a synthesis algorithm based on explicit solution of the LPV-MPC problem is proposed. Another approach based on successive linearisations is proposed in [8]. Here, it is assumed that the longitudinal speed is slowly varying and could be considered constant over the prediction horizon (here $N_{p} \times T_{s}=70 \mathrm{~ms}$ ). Then, the LPV-MPC problem is reduced to the solution of a standard LMPC problem at each sampling time. This formulation allows to consider the effect of the varying longitudinal speed on the lateral guidance. Thus, the interconnected control architecture presented in Figure 6 allows to handle the problem of a coupled longitudinal/lateral control.

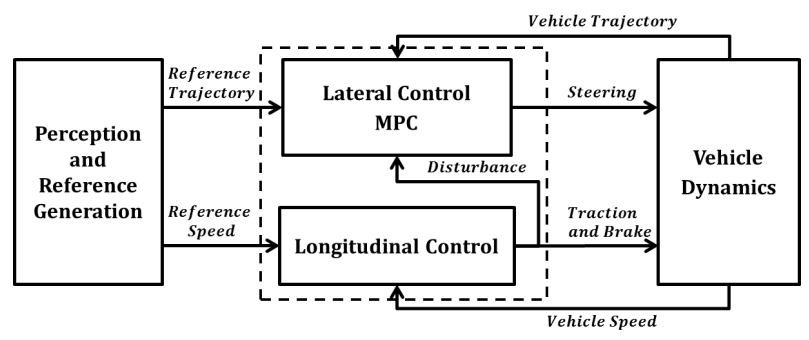

FIGURE 6: Coupled longitudinal and lateral control.

\section{Tests and Simulations}

The effectiveness of the proposed control design is shown through realistic simulations. Firstly, the longitudinal control strategy is tested for the tracking of time-varying reference speed. After then, the automated driving through defined trajectory at variable speed is performed using the proposed integrated longitudinal/lateral guidance architecture.

\subsection{Simulation Conditions}

The validation of the longitudinal control is achieved using the full car drivetrain benchmark available in Matlab/Simulink [29]. In this way, the synthesis model previously presented is not the same as the validation model. Indeed, the 
validation model considers an engine map as well as a mechanical modelling of the driveline and the gearbox as it can be seen in Figure 7 .

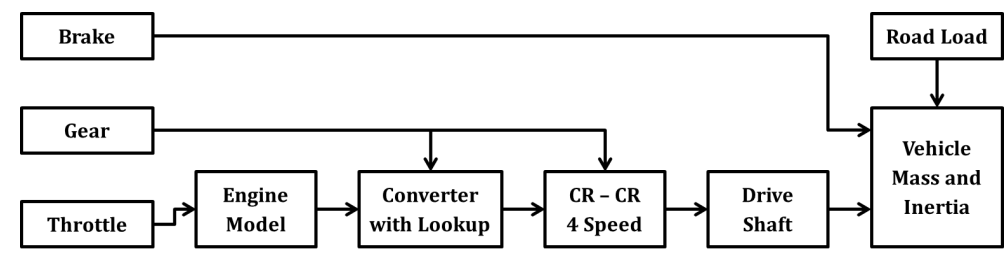

FIGURE 7: Vehicle longitudinal simulation model.

For the whole guidance strategy validation, the $2 \mathrm{D}$ nonlinear chassis model with a nonlinear tyre model presented in [2] is employed. Table 1 summarises the main parameters of the longitudinal and lateral models.

\begin{tabular}{|l|l|c|}
\hline Parameter & Description & Value \\
\hline \hline \multicolumn{2}{|l|}{ Longitudinal model parameters } \\
\hline$C_{\lambda}$ & longitudinal tyre stiffness coefficient & $200000 \mathrm{~N} / \mathrm{rad}$ \\
\hline$C_{a}$ & aerodynamic coefficient & $0.13 \mathrm{~kg} / \mathrm{m}$ \\
\hline$C_{r}$ & rolling resistance coefficient & 150 \\
\hline$g$ & gravity & $9.81 \mathrm{~ms}^{-2}$ \\
\hline$I_{e}$ & engine inertia & $1 \mathrm{kgm}^{2}$ \\
\hline$I_{w}$ & wheel inertia & $0.9 \mathrm{kgm}^{2}$ \\
\hline$K_{t c}$ & K-factor & 30 \\
\hline$R_{f}$ & final drive ratio & 3.28 \\
\hline$R_{t r}$ & gearbox ratio & $17: 2.34,2: 1.45,3: 1.00,4: 0.68]$ \\
\hline \hline Linear bicycle model parameters & $1700 \mathrm{~kg}$ \\
\hline$m$ & vehicle mass & $3048 \mathrm{kgm}$ \\
\hline$I_{z}$ & moment of inertia & $1.04 \mathrm{~m}$ \\
\hline$l_{f}$ & front CoG-distance & $1.6 \mathrm{~m}$ \\
\hline$l_{r}$ & rear CoG-distance & $105700 \mathrm{~N} / \mathrm{rad}$ \\
\hline$C_{f}$ & front cornering stiffness coefficient & $79000 \mathrm{~N} / \mathrm{rad}$ \\
\hline$C_{r}$ & rear cornering stiffness coefficient & \\
\hline
\end{tabular}

TABLE 1: Parameters of the longitudinal and lateral models. 


\subsection{Parameters of the Controllers and Stability Analysis}

The parameters of the longitudinal and lateral controllers are given in Table 2. A solution satisfying the a posteriori exponential stability condition given by the LMI (42) is computed using the SeDuMi solver [30] and the Yalmip interface [13]. For a given decay rate $\alpha=4.7$, the matrix $P$ obtained is :

$$
P=\left[\begin{array}{ccc}
0.0141 & 0.0181 & -0.0000 \\
0.0181 & 0.0886 & 0.0004 \\
-0.0000 & 0.0004 & 1.3650
\end{array}\right]
$$

For a maximum decay rate $\alpha=3.95$, a solution satisfying the a posteriori robust exponential stability condition (48) is given :

$$
P=\left[\begin{array}{ccc}
0.0141 & 0.0141 & -0.0001 \\
0.0141 & 0.1024 & 0.0001 \\
-0.0001 & 0.0001 & 0.9528
\end{array}\right]
$$

with the attenuation level $\gamma=0.9074$. Consequently, the impact of the virtual disturbance $d$ on the tracking error vector $e$ is attenuated. Here, the value considered for $\alpha$ is maximum value satisfying (48). Note that the attenuation level can be decreased considering a lower decay rate $\alpha$.

\begin{tabular}{|l|l|c|}
\hline Parameter & Description & Value \\
\hline \hline \multicolumn{2}{|l|}{ Longitudinal controller parameters } \\
\hline$k_{v}$ & decay rate & 70 \\
\hline$k_{\lambda}$ & decay rate & 35 \\
\hline$k_{T}$ & decay rate & 5 \\
\hline \hline \multicolumn{2}{|l|}{ Lateral controller parameters } \\
\hline$Q$ & error tracking weighting matrix & {$\left[\begin{array}{ll}1 & 0 \\
0 & 1\end{array}\right]$} \\
\hline$R$ & control input weighting matrix & 0.05 \\
\hline$N_{p}$ & prediction horizon & 7 \\
\hline$N_{c}$ & control horizon & 4 \\
\hline$T_{S}$ & sampling time & $10 \mathrm{~ms}$ \\
\hline
\end{tabular}

TABLE 2: Controller parameters.

The sampling time $T_{s}=10 \mathrm{~ms}$ is a common choice in the vehicle control framework and depends on the implementation technology. The choice of the MPC 
parameters $N_{p}, N_{c}, Q$ and $R$ has been fixed through a trial and error approach. To the best knowledge of the authors, there is not a systematic method to choose $a$ priori the MPC parameters ensuring stability and desired performance, particularly in trajectory tracking problems. The stability analysis of the MPC scheme goes beyond the scope of this paper (details on stability analysis for MPC can be found in [19]).

\subsection{Longitudinal Control Test}

The proposed longitudinal control is tested in tracking different reference speeds in acceleration and deceleration phases. The speed tracking results are shown in Figure 8(a). The tracking of the reference speed in acceleration and deceleration phases is realised through throttle and brake. Figure 8(b) shows the evolution of the action on the throttle and brake. The speed tracking is more effective in deceleration phases owing to the high braking capabilities. Figure 8(c) shows the evolution of the engine speed, it can be noted that the engine speed remains in an acceptable operating range $[2000 \mathrm{rpm}, 3000 \mathrm{rpm}]$ thanks to the adopted gear shift policy, see Section 2.4. Note that the vehicle speed is regulated at different values to highlight the gear shifts at time instants $\{21.3 s, 31.8 s, 70.4 s\}$, as illustrated in Figure 8(d).

Figure 9 presents comparative simulations between the control strategy proposed here and the control approach given in [3] respectively named controller 1 and controller 2. The reference speed profile and the vehicle response using both controllers are shown in Figure 9(a). The throttle opening and brake activation are given in Figures 9(c) and 9(d). Controller 1 shows more effective speed-tracking as shown in Figure 9(b). Note that, between $50 s$ and $60 s$ a resistive gravitational force acting as an external disturbance is applied. The magnitude of this disturbance represents a $10 \%$ positive slope. Figure 9(b) shows also a better perturbation rejection using the proposed control strategy.

\subsection{Coupled Longitudinal and Lateral Test}

For the whole guidance architecture tests the reference trajectory to be tracked by the vehicle is obtained from reference generation level using real-world GPS/Cartography data, further details on the reference generation aspects are available in [1]. The fully automated vehicle guidance is performed through an integrated longitudinal and lateral control strategy. The effectiveness of the whole control strategy is shown by performing path and speed tracking on a given trajectory calculated from real-world data. The road geometry coordinates are calculated based on numerical map data and the reference speed profile is generated 


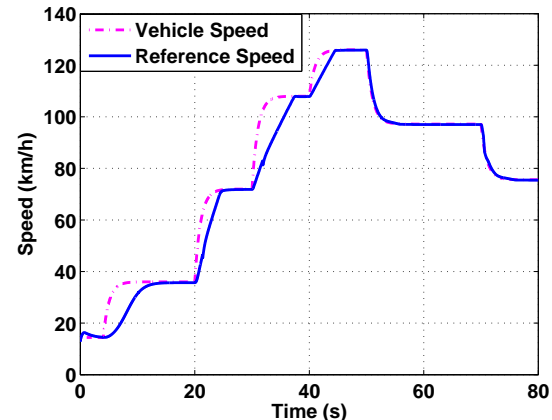

(a) Reference and vehicle speeds

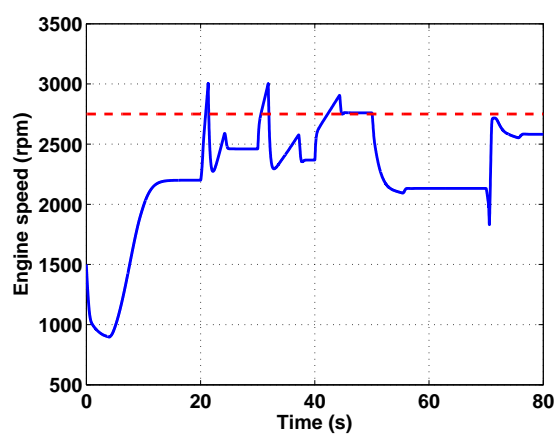

(c) Engine speed

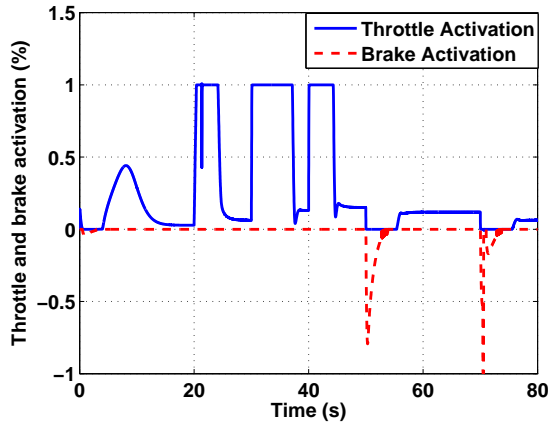

(b) Throttle and brake switching

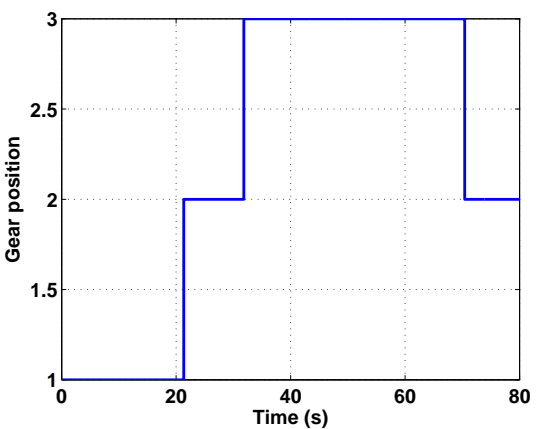

(d) Gear shifts

FIGURE 8: Longitudinal control simulation results. 


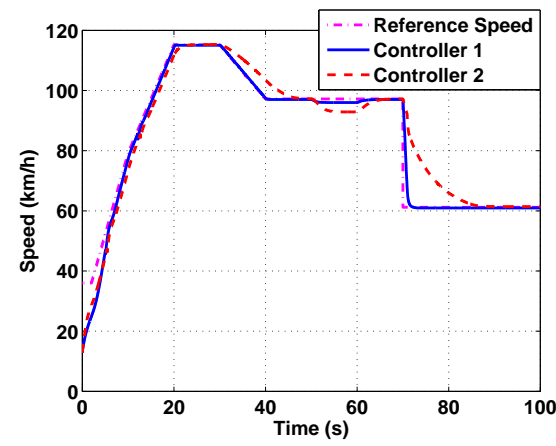

(a) Reference and vehicle speeds

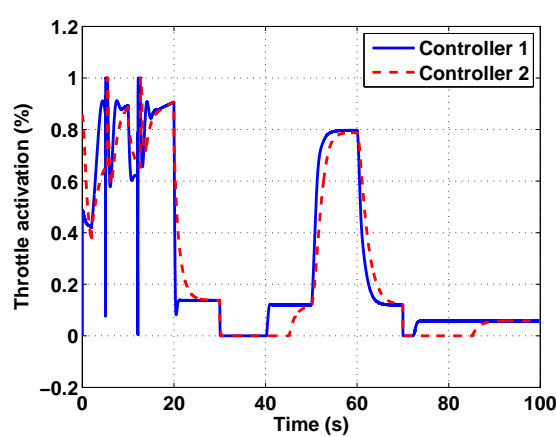

(c) Throttle activation

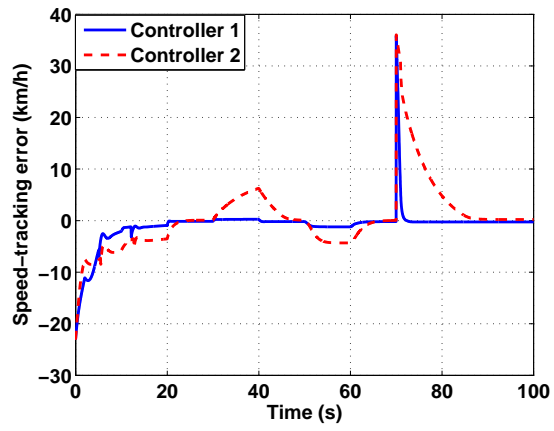

(b) Speed-tracking errors

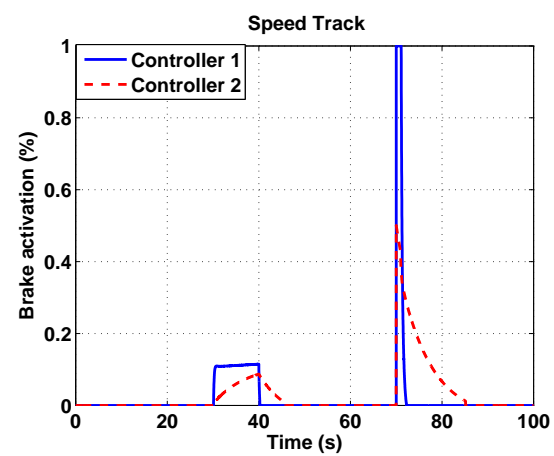

(d) Brake activation

FIGURE 9: Comparative simulation results. 
considering safety and comfort criteria as discussed in [2]. Figure 10(b) shows the reference and the vehicle trajectories. The lateral position as well as the heading angle errors are shown in Figure 10(c). It can be noticed that the tracking errors are very acceptable. The speed tracking results are shown in Figure 10(d). Remark that the test trajectory includes different driving situations, first on a highway at $110 \mathrm{~km} / \mathrm{h}$, then in the highway exit where an important lateral solicitation at reduced speed $(20 \mathrm{~km} / \mathrm{h}$ to $50 \mathrm{~km} / \mathrm{h})$ is performed and finally an acceleration phase to reach the speed of $90 \mathrm{~km} / \mathrm{h}$ on a rural road. Thanks to the proposed integrated longitudinal/lateral control strategy, the fully automated guidance of the vehicle is evaluated along a typical road.

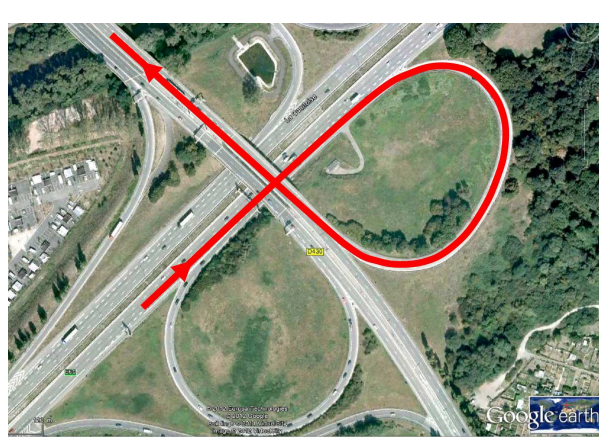

(a) Highway exit map

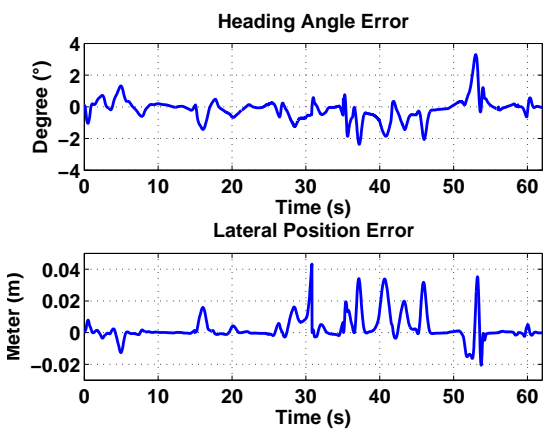

(c) Lateral position and heading errors

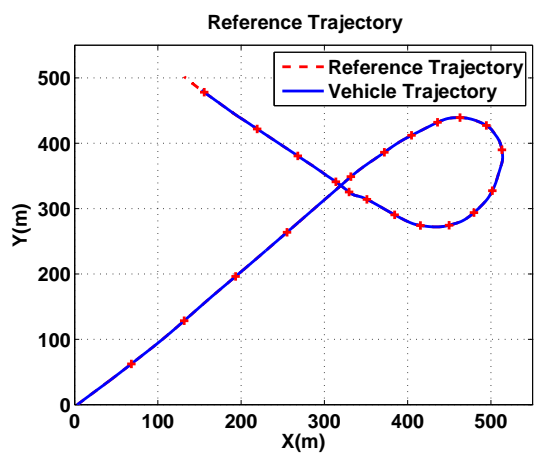

(b) Reference and vehicle trajectories

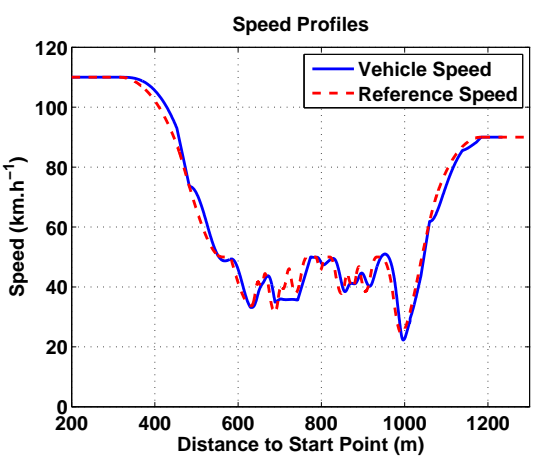

(d) Reference speed tracking

FIGURE 10: Combined longitudinal and lateral control test. 


\section{Conclusions and Perspectives}

Energy Consumption Reduction Discussion. The objective of the proposed control strategy, in both longitudinal or longitudinal/lateral dimensions, is to provide global and effective vehicle guidance strategies that could be extended to take fuel consumption aspects into account. The energy management can be introduced at different layers in the guidance architecture proposed here. The first way is to introduce energy consumption criteria at the reference generation layer. In fact, the generation of smooth reference speed profiles helps to guarantee eco-friendly driving and contributes to reduce energy consumption. In this paper, the reference speed generation is done without considering road slope variations. The authors aim to generalize the approach proposed in [7] by considering 3D cartography and trajectory generation. The proposed longitudinal control allows to handle this additional information since the rolling resistance forces are considered in controller design. The second way is to improve the torque control by optimizing the gear sequence. The assumption made in this work allows to keep the engine speed in an acceptable range not far from the optimal operating point. These aspects are investigated in the literature through optimization approach. In [16] the problem of minimizing fuel consumption on a known road part using a dynamic programming approach is proposed. A look-ahead strategy is introduced by [9] and the problem is solved using the same technique i.e. dynamic programming. Indeed, the problem of choosing the appropriate gear ratio to meet some energetic cost is a mixed integer optimization problem for which real-time solution risks to be hard. In future work, the authors aim to introduce energy management criteria following a predictive approach using information provided by the perception level.

Conclusion. In this paper, a longitudinal control design based on nonlinear cascade control is proposed. The cascade loop ensures reference speed tracking and torque control. The longitudinal controller is coupled with a lateral controller in an interconnected architecture to ensure fully automated driving. The lateral controller is based on a model predictive control design. Through simulations, the effectiveness of the longitudinal controller in tracking time-varying reference speed is shown. The automated guidance strategy is tested using real-world trajectory data and showed promising results.

\section{Références}

[1] R. Attia, J. Daniel, J-P. Lauffenburger, R. Orjuela, and M. Basset. Reference Generation and Control Strategy for Automated Vehicle Guidance. In IEEE Intelligent Vehicle Symposium (IV'12), Alcalá de Henares, Spain, 2012. 
[2] R. Attia, R. Orjuela, and M. Basset. Coupled Longitudinal and Lateral Control Strategy Improving Lateral Stability for Autonomous Vehicle. In American Control Conference (ACC'12), Montreal, Canada, 2012.

[3] R. Attia, R. Orjuela, and M. Basset. Longitudinal Control for Automated Vehicle Guidance. In IFAC Workshop on Engine and Powertrain Control, Simulation and Modeling (E-COSM'12), Rueil-Malmaison, France, 2012.

[4] T. Besselmann. Constrained Optimal Control of Piecewise Affine and Linear Parameter-Varying Systems. PhD thesis, ETH Zürich, 2010.

[5] T. Besselmann and M. Morari. Autonomous Vehicle Steering Using Explicit LPV-MPC. In European Control Conference (ECC'09), Budapest, Hungary, 2009.

[6] S. Boyd, L. El Ghaoui, E. Feron, and V. Balakrishnan. Linear Matrix Inequalities in System and Control Theory. Society for Industrial and Applied Mathematics, 1994.

[7] J. Daniel, A. Birouche, J-P. Lauffenburger, and M. Basset. Navigationbased Constrained Trajectory Generation for Advanced Driver Assistance Systems. International Journal of Vehicle Autonomous Systems (IJVAS), 9 :269-296, 2011.

[8] P. Falcone, F. Borrelli, J. Asgari, H. E. Tseng, and D. Hrovat. Predictive Active Steering Control for Autonomous Vehicle Systems. IEEE Trans. on Control Systems Technology, 15 :566-580, 2007.

[9] L. Guzzella. Automobiles of the Future and the Role of Automatic Control in those Systems. Annual Reviews in Control, $33: 1-10,2009$.

[10] L. Guzzella and A. Sciarretta. Vehicle Propulsion Systems. Springer-Verlag, 2005.

[11] T. Keviczky, P. Falcone, F. Borrelli, J. Asgari, and D. Hrovat. Predictive control approach to autonomous vehicle steering. In American Control Conference (ACC'06), Minnesota, USA, 2006.

[12] U. Kiencke and L. Nielsen. Automotive Control Systems. Springer-Verlag, 2000 . 
[13] J. Löfberg. YALMIP : a Toolbox for Modeling and Optimization in Matlab. In Computer Aided Control Systems Design (CACSD), Taipei, Taiwan, 2004.

[14] E. M. Lim. Lateral and Longitudinal Vehicle Control Coupling in the Automated Highway System. Master's thesis, University of California at Berkeley, 1998.

[15] X-Y. Lu and J. K. Hedrick. Impact of Combined Longitudinal, Lateral and Vertical Control on Autonomous Road Vehicle Design. Int. J. Vehicle Autonomous Systems, 2 :40-70, 2004.

[16] H-T. Luu, L. Nouvelière, and S. Mammar. Dynamic Programming for Fuel Consumption Optimization on Light Vehicle. In 6th IFAC Symposium Advances in Automotive Control, Holiday Inn Munich Schwabing, Germany, 2010.

[17] E. Maalouf, M. Saad, and H. Saliah. A Higher Level Path Tracking Controller for a Four-wheel Differentially Steered Mobile Robot. Robotics and Autonomous Systems, 54 :23-33, 2006.

[18] K. El Majdoub, F. Giri, H. Ouadi, L. Dugard, and F.Z. Chaoui. Vehicle Longitudinal Modeling for Nonlinear Control. Control Engineering Practice, $20: 69-81,2012$.

[19] D.Q. Mayne, J.B. Rawlings, C.V. Rao, and P.O. Scokaert. Constrained Model Predictive Control : Stability and Optimality. Automatica, 36 :789-814, 2000 .

[20] L. Menhour, B. d'Andréa Novel, C. Boussard, M. Fliess, and H. Mounier. Algebraic Nonlinear Estimation and Flatness-based Lateral/Longitudinal Control for Automotive Vehicles. In International Conference on Intelligent Transportation Systems (ITSC'11), Washington, DC, USA, 2011.

[21] L. Nehaoua and L. Nouvelière. Backstepping Based Approach for the Combined Longitudinal-Lateral Vehicle Control. In IEEE Intelligent Vehicle Symposium (IV'12), Alcala de Henares, Spain, 2012.

[22] L. Nouvelière and S. Mammar. Experimental Vehicle Longitudinal Control Using a Second Order Sliding Mode Technique. Control Engineering Practice, 15 :943-954, 2007. 
[23] E. Onieva, J.E.Naranjo, V. Milanés, J. Alonso, R. Garcia, and J. Pérez. Automatic Lateral Control for Unmanned Vehicles via Genetic Algorithms. Applied Soft Computing, 11 :1303-1309, 2010.

[24] R. Rajamani and S. E. Shladover. An Experimental Comparative Study of Autonomous and Co-operative Vehicle-Follower Control Systems. Transportation Research Part C, 9 :15-31, 2001.

[25] L. Re, F. Allgöwer, L. Glielmo, C. Guardiola, and I. Kolmanovsky. Automotive Model Predictive Control. Springer-Verlag, 2010.

[26] W. J. Rugh and J. S. Shamma. Research on Gain Scheduling. Automatica, $36: 1401-1425,2000$.

[27] P. Shakouri, A. Ordys, and M. Askari. Adaptive Cruise Control with Stop\&Go Function Using the State-dependent Nonlinear Model Predictive Control Approach. ISA Transactions, 51 :622-631, 2012.

[28] P. Shakouri, A. Ordys, M. Askari, and D. S. Laila. Adaptive Cruise Control System : Comparing Gain-Scheduling PI and LQ Controllers. In 18th IFAC World Congress, Milano, Italy, 2010.

[29] P. Shakouri, A. Ordys, M. Askari, and D. S. Laila. Longitudinal Vehicle Dynamics Using Matlab/Simulink. In UKACC International Conference on Control, Coventry, UK, 2010.

[30] J. F. Sturm. Using SeDuMi 1.02, A Matlab Toolbox for Optimization over Symmetric Cones. Optimization Methods and Software, 11 :625-653, 1999. 